Research Article

\title{
A 3-D Novel Conservative Chaotic System and its Generalized Projective Synchronization via Adaptive Control
}

\author{
S. Vaidyanathan*, ${ }^{1}$ and S. Pakiriswamy ${ }^{2}$ \\ ${ }^{I}$ Research and Development Centre, Vel Tech University, Avadi, Chennai-600062, Tamil Nadu, India. \\ ${ }^{2}$ School of Information and Computing Technology, Vel Tech University, Avadi, Chennai-600062, Tamil Nadu, India.
}

Received 30 September 2014; Revised 14 November 2014; Accepted 25 November 2014

\begin{abstract}
This research work proposes a five-term 3-D novel conservative chaotic system with a quadratic nonlinearity and a quartic nonlinearity. The conservative chaotic systems have the important property that they are volume conserving. The Lyapunov exponents of the 3-D novel chaotic system are obtained as $L_{1}=0.0836, L_{2}=0$ and $L_{3}=-0.0836$. Since the sum of the Lyapunov exponents is zero, the 3-D novel chaotic system is conservative. Thus, the Kaplan-Yorke dimension of the 3-D novel chaotic system is easily seen as 3.0000. The phase portraits of the novel chaotic system simulated using MATLAB depict the chaotic attractor of the novel system. This research work also discusses other qualitative properties of the system. Next, an adaptive controller is designed to achieve Generalized Projective Synchronization (GPS) of two identical novel chaotic systems with unknown system parameters. MATLAB simulations are shown to validate and demonstrate the GPS results derived in this work.
\end{abstract}

Keywords: Chaos, chaotic systems, conservative systems, synchronization, generalized projective synchronization, adaptive control.

\section{Introduction}

There is great interest in the chaos literature in the discovery of chaotic behaviour in nature and physical systems. Chaotic systems are defined as nonlinear dynamical systems which are very sensitive to initial conditions, topologically mixing and also with dense periodic orbits [1].

A significant development in chaos theory occurred when Lorenz discovered a 3-D chaotic system of a weather model [2]. This was followed by the discovery of many 3-D chaotic systems in the chaos literature such as Rössler system [3], Rabinovich system [4], ACT system [5], Sprott systems [6], Chen system [7], Lü system [8], Shaw system [9], Feeny system [10], Shimizu system [11], Liu-Chen system [12], Cai system [13], Tigan system [14], Colpitt's oscillator [15], WINDMI system [16], Zhou system [17], etc.

Recently, many 3-D chaotic systems have been discovered such as Li system [18], Elhadj system [19], Pan system [20], Sundarapandian system [21], Yu-Wang system [22], Sundarapandian-Pehlivan system [23], Zhu system [24], Vaidyanathan systems [25-30], VaidyanathanMadhavan system [31], Pehlivan-Moroz-Vaidyanathan system [32], Jafari system [33], Pham system [34], etc.

We note that the chaotic systems [2-34] are dissipative systems in which the system limit sets are ultimately confined into a specific limit set of zero volume, and the asymptotic motion of the chaotic system settles onto a strange attractor of the system.

In the chaos literature, there is also an active interest in the discovery of conservative chaotic systems [35], which have the special property that the volume of the flow is conserved.

A classical example of a conservative chaotic system is the Nosé-Hoover system $[36,37]$, which is modelled by the system of differential equations

$$
\left\{\begin{array}{l}
\frac{d x_{1}}{d t}=x_{2} \\
\frac{d x_{2}}{d t}=-x_{1}+x_{2} x_{3} \\
\frac{d x_{3}}{d t}=1-x_{2}^{2}
\end{array}\right.
$$

The Nosé-Hoover system (1) has the Lyapunov exponents $L_{1}=0.014, L_{2}=0$ and $L_{3}=-0.014$. The system (1) is chaotic as it has a positive Lyapunov exponent and is conservative as the sum of the Lyapunov exponents is zero. Thus, the system (1) is volume-conserving.

* E-mail address: sundarvtu@gmail.com ISSN: 1791-2377 @ 2015 Kavala Institute of Technology. All rights reserved. 
Also, the Kaplan-Yorke dimension of the Nosé-Hoover system (1) is easily seen as

$D_{K Y}=2+\frac{L_{1}+L_{2}}{\left|L_{3}\right|}=2+1=3$

In this research work, we modify the dynamics of NoséHoover chaotic system (1) and obtain a 3-D novel conservative chaotic system with Lyapunov exponents $L_{1}=0.0836, L_{2}=0$ and $L_{3}=-0.0836$. Thus, it is clear that the maximal Lyapunov exponent (MLE) of the novel conservative chaotic system is $L_{1}=0.0836$, which is greater than the maximal Lyapunov exponent (MLE) of the NoséHoover chaotic system (1). The Kaplan-Yorke dimension of all 3-D conservative chaotic systems is equal to three.

The study of chaos theory has many important applications in science and engineering such as oscillators [38-40], lasers [41-43], robotics [44-47], chemical reactors [48-50], biology [51,52], ecology [53,54], cardiology [55], memristors [56-60], neural networks [61-63], secure communications [64-67], cryptosystems [68-71], economics [72-74], etc.

Chaos control and chaos synchronization are important research problems in the chaos theory. In the last three decades, many mathematical methods have been developed successfully to address these research problems.

The study of control of a chaotic system investigates methods for designing feedback control laws that globally or locally asymptotically stabilize or regulate the outputs of a chaotic system.

Many methods have been developed for the control and tracking of chaotic systems such as active control [75-78], adaptive control [79-85], backstepping control [86-88], sliding mode control $[89,90]$, etc.

Chaos synchronization problem deals with the synchronization of a couple of systems called the master or drive system and the slave or response system. To solve this problem, control laws are designed so that the output of the slave system tracks the output of the master system asymptotically with time.

Because of the butterfly effect, this is a challenging problem even when the initial conditions of the master and slave systems are nearly identical because of the exponential divergence of the outputs of the two systems in the absence of any control. The synchronization of chaotic systems has applications in secure communications [91-93], cryptosystems [94, 95], encryption [96, 99], etc.

In the chaos literature, many different methodologies have been also proposed for the synchronization and antisynchronization of chaotic systems such as PC method [100], active control [101-111], time-delayed feedback control [112,113], adaptive control [114-125], sampled-data feedback control [126-129], backstepping control [130-136], sliding mode control [137-143], etc.

Furthermore, we derive an adaptive control law that achieves generalized projective synchronization (GPS) of the identical 3-D novel conservative chaotic systems when the system parameters are unknown. Generalized projective synchronization is a general type of synchronization which generalizes complete synchronization, anti-synchronization, hybrid synchronization, and projective synchronization of chaotic systems. The main synchronization result is proved using adaptive control theory and Lyapunov stability theory. MATLAB simulations are shown in detail to validate and demonstrate the generalized projective synchronization of the identical 3-D novel conservative chaotic systems.

\section{A Five-Term 3-D Novel Conservative Chaotic System}

The dynamics of the five-term novel 3-D conservative chaotic system is described by

$$
\left\{\begin{array}{l}
\frac{d x_{1}}{d t}=x_{2} \\
\frac{d x_{2}}{d t}=-x_{1}+a x_{2} x_{3} \\
\frac{d x_{3}}{d t}=b-x_{2}^{4}
\end{array}\right.
$$

where $x_{1}, x_{2}, x_{3}$ are the states and $a, b$ are positive parameters.

The nonlinear system (3) depicts a chaotic attractor when the parameter values are taken as:

$a=1, b=1$

We take the initial conditions as

$x_{1}(0)=0.2, x_{2}(0)=0.2, x_{3}(0)=0.2$

The 3-D portrait of the strange chaotic attractor (3) for the parameter values (2) and the initial conditions (5) is depicted in Fig. 1, and the 2-D portraits (projections on the three coordinate planes) are depicted in Figs. 2-4.

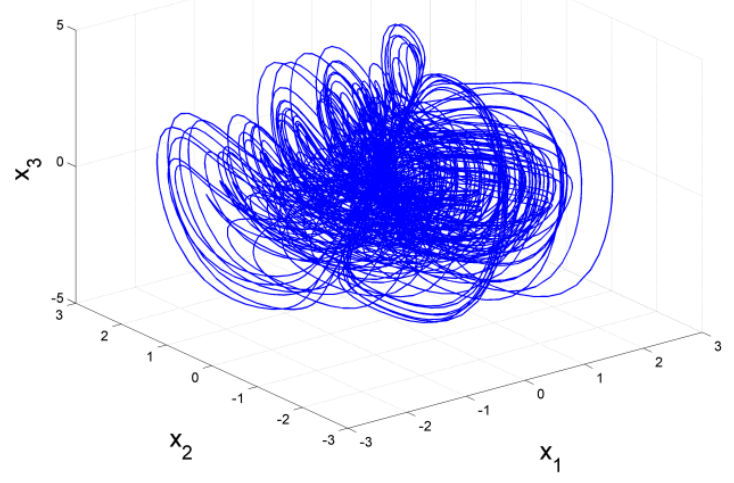

Fig. 1. The chaotic attractor of the novel chaotic system in $R^{3}$.

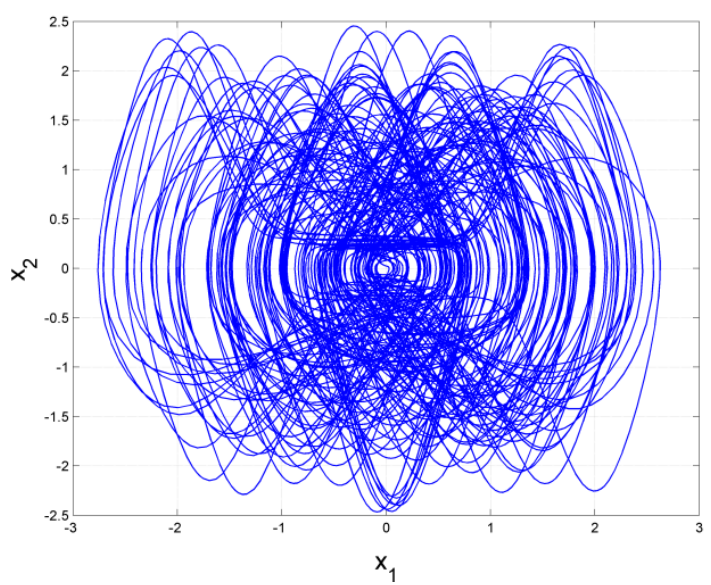

Fig. 2. The 2-D projection of the chaotic attractor on the $\left(x_{1}, x_{2}\right)$ plane. 


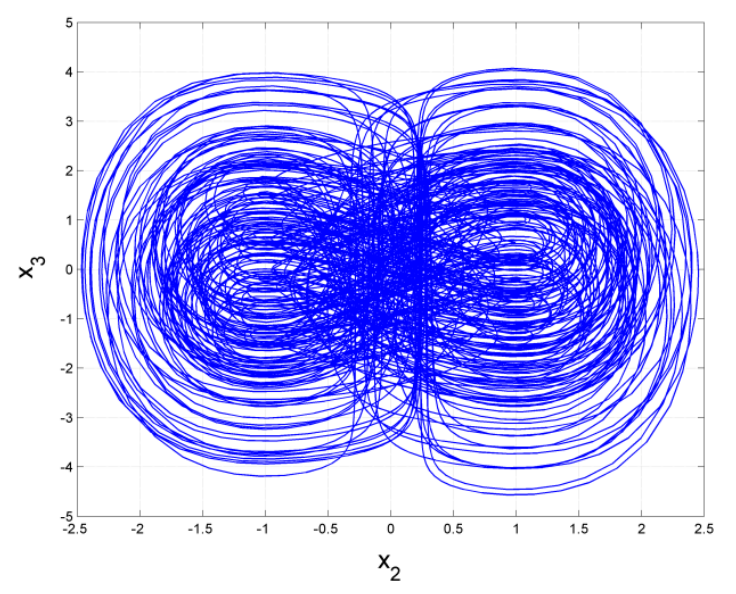

Fig. 3. The 2-D projection of the chaotic attractor on the $\left(x_{2}, x_{3}\right)$ plane.

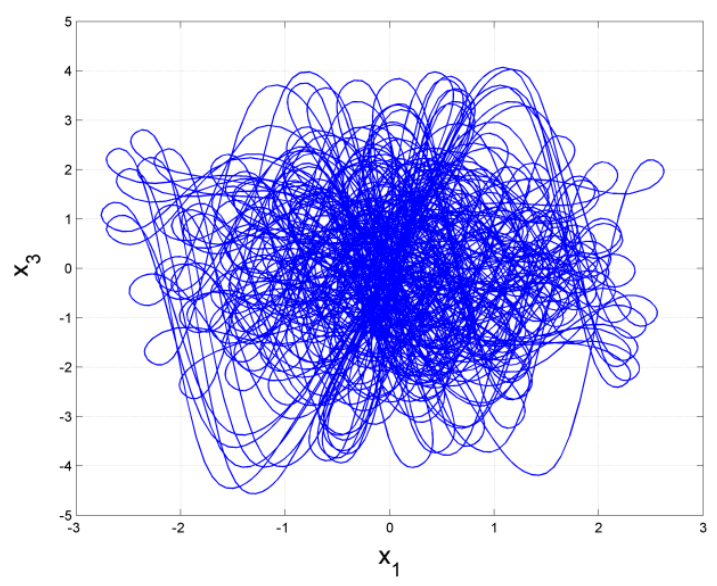

Fig. 4. The 2-D projection of the chaotic attractor on the $\left(x_{1}, x_{3}\right)$ plane.

\section{Analysis of the 3-D Novel Chaotic System}

In this section, qualitative properties of the 3-D novel chaotic system (3) are detailed.

\subsection{Volume Conservation of the Flow}

In vector notation, we may express the system (3) as:

$\frac{d x}{d t}=f(\boldsymbol{x})=\left[\begin{array}{l}f_{1}\left(x_{1}, x_{2}, x_{3}\right) \\ f_{2}\left(x_{1}, x_{2}, x_{3}\right) \\ f_{3}\left(x_{1}, x_{2}, x_{3}\right)\end{array}\right]$

where

$\left\{\begin{array}{l}f_{1}\left(x_{1}, x_{2}, x_{3}\right)=x_{2} \\ f_{2}\left(x_{1}, x_{2}, x_{3}\right)=-x_{1}+a x_{2} x_{3} \\ f_{3}\left(x_{1}, x_{2}, x_{3}\right)=b-x_{2}^{4}\end{array}\right.$

We take the parameter values as in the chaotic case, viz. $a=1$ and $b=1$.

Let $\Omega$ be any region in $\boldsymbol{R}^{3}$ with a smooth boundary and also $\Omega(t)=\Phi_{t}(\Omega)$, where $\Phi_{t}$ is the flow of $f$.

Furthermore, let $V(t)$ denote the volume of $\Omega(t)$.

By Liouville's theorem, we have $\frac{d V}{d t}=\int_{\Omega(t)}(\nabla \cdot f) d x_{1} d x_{2} d x_{3}$

The divergence of the novel chaotic system (3) is easily found as:

$\nabla \cdot f=\frac{\partial f_{1}}{\partial x_{1}}+\frac{\partial f_{2}}{\partial x_{2}}+\frac{\partial f_{3}}{\partial x_{3}}=0+0+0=0$

Substituting (9) into (8), we obtain the first order ODE

$\frac{d V}{d t}=0$

Integrating (10), we obtain the unique solution as:

$V(t)=V(0) \quad$ for all $t \geq 0$

This shows that the 3-D novel chaotic system (3) is volume-conserving. Hence, the system (3) is a conservative chaotic system.

\subsection{Symmetry and Invariance}

It is easy to see that the system (3) is invariant under the coordinates transformation

$$
\left(x_{1}, x_{2}, x_{3}\right) \mapsto\left(-x_{1},-x_{2}, x_{3}\right)
$$

Thus, the system (3) has rotation symmetry about the $x_{3}$-axis and any non-trivial trajectory of the system (3) must have a twin trajectory. It is also easy to see that the $x_{3}$-axis is invariant under the flow of the system (3).

\subsection{Equilibrium Points}

The equilibrium points of the novel chaotic system (3) are obtained by solving the following system of equations (with $a=1$ and $b=1$ )

$$
\left\{\begin{aligned}
x_{2} & =0 \\
-x_{1}+a x_{2} x_{3} & =0 \\
b-x_{2}^{4} & =0
\end{aligned}\right.
$$

Since the first and last equations of the system (13) contradict each other, it is immediate that the system (13) does not admit any solution. Hence, there is no equilibrium for the novel chaotic system (3).

\subsection{Lyapunov Exponents and Kaplan-Yorke Dimension}

For the chosen parameter values (4), the Lyapunov exponents of the novel chaotic system (3) are obtained using MATLAB as:

$L_{1}=0.0836, L_{2}=0, L_{3}=-0.0836$

Since the spectrum of Lyapunov exponents (14) has a positive term $L_{1}$, it follows that the 3-D novel system (1) is chaotic. Since the sum of the Lyapunov exponents is zero, the novel chaotic system is conservative.

The maximal Lyapunov exponent (MLE) of the novel chaotic system (3) is $L_{1}=0.0836$. 
Also, the Kaplan-Yorke dimension of the novel chaotic system (3) is calculated as:

$$
D_{K Y}=2+\frac{L_{1}+L_{2}}{\left|L_{3}\right|}=2+1=3
$$

Fig. 5 depicts the dynamics of the Lyapunov exponents of the novel chaotic system (3).

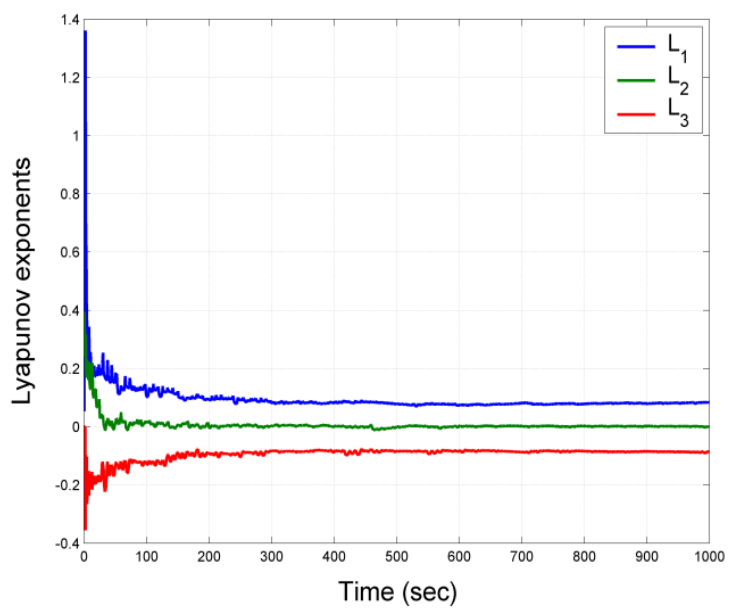

Fig. 5. Dynamics of the Lyapunov Exponents of the Novel System.

\section{Generalized Projective Synchronization of the Identical 3-D Novel Conservative Chaotic Systems}

In this section, new results are derived for an adaptive controller to achieve generalized projective synchronization (GPS) of the identical 3-D novel conservative chaotic systems.

As the master system, we take the novel chaotic system

$$
\left\{\begin{array}{l}
\frac{d x_{1}}{d t}=x_{2} \\
\frac{d x_{2}}{d t}=-x_{1}+a x_{2} x_{3} \\
\frac{d x_{3}}{d t}=b-x_{2}^{4}
\end{array}\right.
$$

where $x_{1}, x_{2}, x_{3}$ are state variables and $a, b$ are unknown, constant, parameters of the system.

As the slave system, we take the novel chaotic system

$$
\left\{\begin{array}{l}
\frac{d y_{1}}{d t}=y_{2}+u_{1} \\
\frac{d y_{2}}{d t}=-y_{1}+a y_{2} y_{3}+u_{2} \\
\frac{d y_{3}}{d t}=b-y_{2}^{4}+u_{3}
\end{array}\right.
$$

where $y_{1}, y_{2}, y_{3}$ are state variables and $u_{1}, u_{2}, u_{3}$ are adaptive controllers to be designed.

The generalized projection synchronization (GPS) error between the identical chaotic systems is defined as:

$$
\left\{\begin{array}{l}
e_{1}(t)=y_{1}(t)-\eta_{1} x_{1}(t) \\
e_{2}(t)=y_{2}(t)-\eta_{2} x_{2}(t) \\
e_{3}(t)=y_{3}(t)-\eta_{3} x_{3}(t)
\end{array}\right.
$$

In (18), $\eta_{1}, \eta_{2}, \eta_{3}$ are real, scaling constants, which are known. (Note that $\eta_{\mathrm{i}}$ can take both positive and negative values).

The GPS error dynamics is calculated as:

$$
\left\{\begin{array}{l}
\frac{d e_{1}}{d t}=y_{2}-\eta_{1} x_{2}+u_{1} \\
\frac{d e_{2}}{d t}=-y_{1}+\eta_{2} x_{1}+a\left(y_{2} y_{3}-\eta_{2} x_{2} x_{3}\right)+u_{2} \\
\frac{d e_{3}}{d t}=b\left(1-\eta_{3}\right)-y_{2}^{4}+\eta_{3} x_{2}^{4}+u_{3}
\end{array}\right.
$$

We consider the adaptive control law

$$
\left\{\begin{aligned}
u_{1}= & -y_{2}+\eta_{1} x_{2}-k_{1} e_{1} \\
u_{2}= & y_{1}-\eta_{2} x_{1}-A(t)\left(y_{2} y_{3}-\eta_{2} x_{2} x_{3}\right) \\
& -k_{2} e_{2} \\
u_{3}= & -B(t)\left(1-\eta_{3}\right)+y_{2}^{4}-\eta_{3} x_{2}^{4}-k_{3} e_{3}
\end{aligned}\right.
$$

where $k_{1}, k_{2}, k_{3}$ are positive gains and $A(t), B(t)$ are estimates of the unknown parameters $a, b$ respectively.

The parameter estimation errors are defined by

$$
\left\{\begin{array}{l}
e_{a}(t)=a-A(t) \\
e_{b}(t)=b-B(t)
\end{array}\right.
$$

Substituting (20) into the error dynamics (19), we get

$$
\left\{\begin{array}{l}
\frac{d e_{1}}{d t}=-k_{1} e_{1} \\
\frac{d e_{2}}{d t}=(a-A(t))\left(y_{2} y_{3}-\eta_{2} x_{2} x_{3}\right)-k_{2} e_{2} \\
\frac{d e_{3}}{d t}=(b-B(t))\left(1-\eta_{3}\right)-k_{3} e_{3}
\end{array}\right.
$$

Using (21), we can simplify the error dynamics (22) as:

$$
\left\{\begin{array}{l}
\frac{d e_{1}}{d t}=-k_{1} e_{1} \\
\frac{d e_{2}}{d t}=e_{a}\left(y_{2} y_{3}-\eta_{2} x_{2} x_{3}\right)-k_{2} e_{2} \\
\frac{d e_{3}}{d t}=e_{b}\left(1-\eta_{3}\right)-k_{3} e_{3}
\end{array}\right.
$$

Differentiating (21) with respect to $t$, we get

$$
\left\{\begin{array}{l}
\frac{d e_{a}}{d t}=-\frac{d A}{d t} \\
\frac{d e_{b}}{d t}=-\frac{d B}{d t}
\end{array}\right.
$$

Next, we use Lyapunov stability theory for finding an update law for the parameter estimates.

Consider the quadratic Lyapunov function defined by

$V=\frac{1}{2}\left(e_{1}^{2}+e_{2}^{2}+e_{3}^{2}+e_{a}^{2}+e_{b}^{2}\right)$

which is positive definite on $R^{5}$.

Differentiating $V$ along the trajectories of (23) and (24), we get

$$
\begin{aligned}
\frac{d V}{d t}= & -k_{1} e_{1}^{2}-k_{2} e_{2}^{2}-k_{3} e_{3}^{2} \\
& +e_{a}\left[e_{2}\left(y_{2} y_{3}-\eta_{2} x_{2} x_{3}\right)-\frac{d A}{d t}\right] \\
& +e_{b}\left[e_{3}\left(1-\eta_{3}\right)-\frac{d B}{d t}\right]
\end{aligned}
$$


In view of (26), we take the parameter update law as:

$$
\left\{\begin{array}{l}
\frac{d A}{d t}=e_{2}\left(y_{2} y_{3}-\eta_{2} x_{2} x_{3}\right) \\
\frac{d B}{d t}=e_{3}\left(1-\eta_{3}\right)
\end{array}\right.
$$

Theorem 1. The adaptive control law (20) and the parameter update law (27) achieve generalized projective synchronization (GPS) between the identical chaotic systems (16) and (17) for all initial conditions, where $k_{1}, k_{2}, k_{3}$ are positive constants.

Proof. We prove this result using Lyapunov stability theory.

For this purpose, we consider the quadratic Lyapunov function $V$ defined by (25), which is positive definite on $R^{5}$.

Substituting the parameter update law (27) into (26), we obtain the time derivative of $V$ as:

$$
\frac{d V}{d t}=-k_{1} e_{1}^{2}-k_{2} e_{2}^{2}-k_{3} e_{3}^{2}
$$

which is a negative semi-definite function on $R^{6}$.

Thus, we can conclude that the synchronization error $e(t)$ and the parameter estimation error are globally bounded.

We define $k=\min \left\{k_{1}, k_{2}, k_{3}\right\}$. Then we get

$$
\frac{d V}{d t} \leq-k\|e\|^{2} \text { or } k\|e\|^{2} \leq-\frac{d V}{d t}
$$

Integrating the inequality (29) from 0 to $t$, we get

$k \int_{0}^{t}\|e(\tau)\|^{2} d \tau \leq V(0)-V(t)$

From (30), it follows that $e(t) \in L_{2}$. Using (23), we can conclude that $\dot{e} \in L_{\infty}$.

Thus, using Barbalat's lemma [140], we conclude that $e(t) \rightarrow 0$ exponentially as $t \rightarrow \infty$ for all initial conditions $e(0)$ $\in R^{3}$. This completes the proof.

For numerical simulations, the parameter values of the novel chaotic systems (16) and (17) are taken as in the chaotic case, viz. $a=1$ and $b=1$. We take the gain constants as $k_{\mathrm{i}}=6$ for $i=1,2,3$. The GPS constants are taken as $\eta_{1}=1.2, \eta_{2}=-0.7$ and $\eta_{3}=1.8$.

The initial conditions of the master system (16) are taken as $x_{1}(0)=9.5, x_{2}(0)=2.3$ and $x_{3}(0)=-6.1$.

The initial conditions of the slave system (17) are taken as $y_{1}(0)=4.8, y_{2}(0)=-2.9$ and $y_{3}(0)=7.5$.

The initial conditions of the parameter estimates are taken as $A(0)=4.2$ and $B(0)=0.2$.

Figs. 6-8 describe the generalized projective synchronization (GPS) of the novel chaotic systems (16) and (17), while Fig. 9 describes the time-history of the synchronization errors $e_{1}, e_{2}, e_{3}$

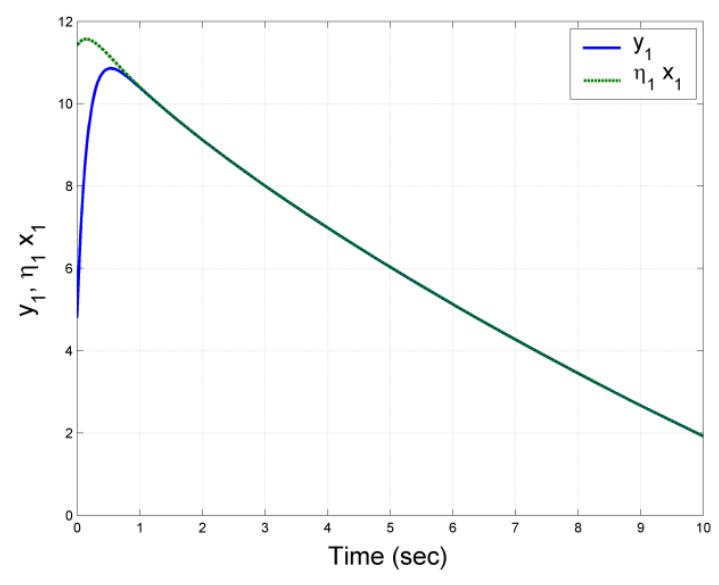

Fig. 6. GPS of the states $x_{1}$ and $y_{1}$ of the novel chaotic systems.

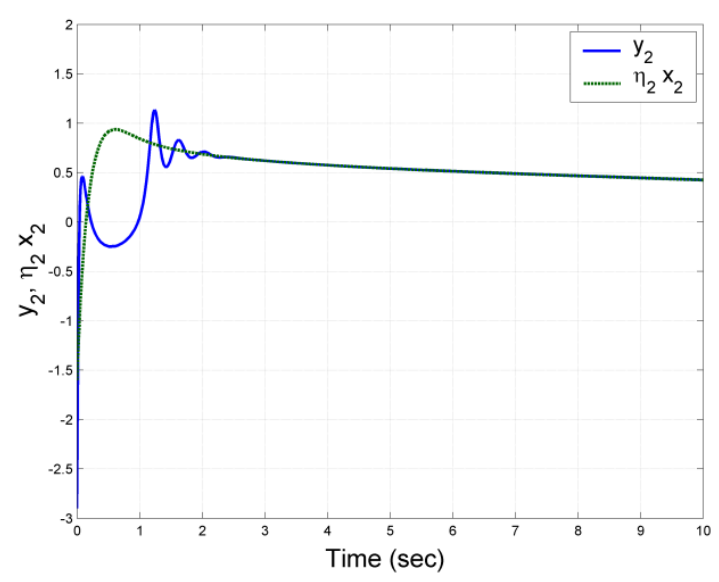

Fig. 7. GPS of the states $x_{2}$ and $y_{2}$ of the novel chaotic systems.

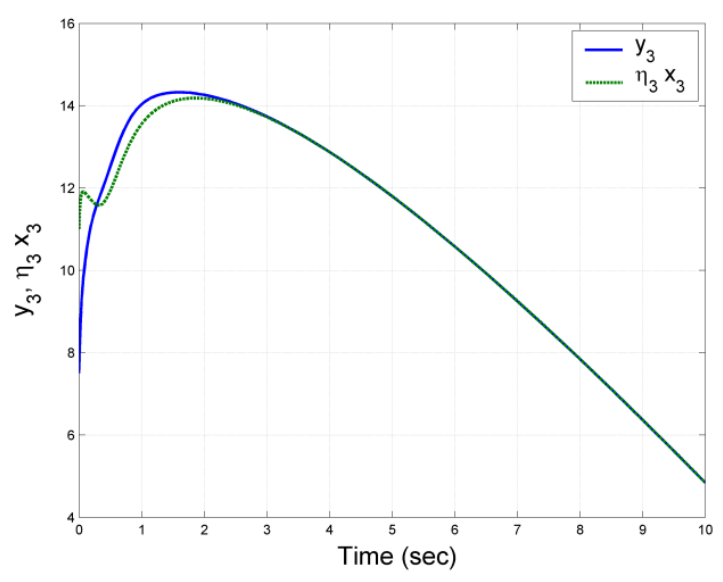

Fig. 8. GPS of the states $x_{3}$ and $y_{3}$ of the novel chaotic systems. 


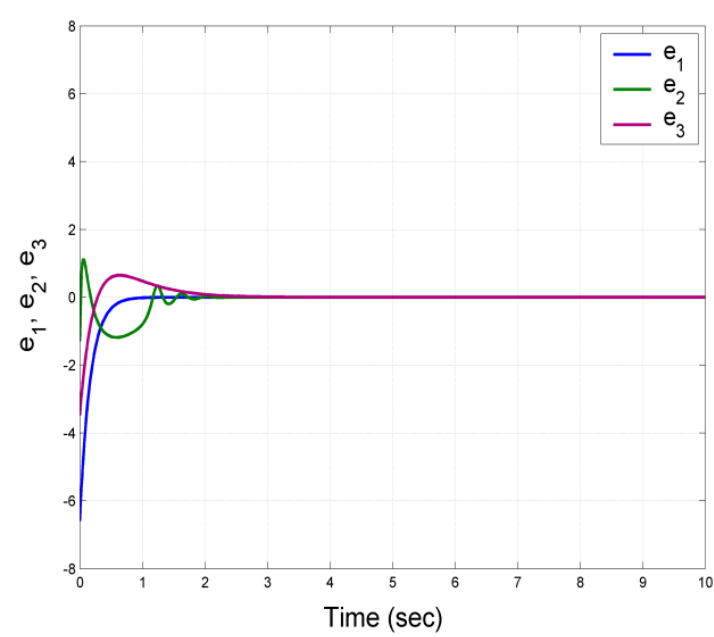

Fig. 9. Time-history of the GPS errors $e_{1}, e_{2}, e_{3}$.

\section{Conclusions}

In this research work, we have proposed a five-term 3-D novel conservative chaotic system with a quadratic nonlinearity and a quartic nonlinearity. The conservative chaotic systems have the important property that they are volume conserving. Also, the Kaplan-Yorke dimension of any 3-D conservative chaotic system is equal to 3 . The Lyapunov exponents of the 3-D novel chaotic system have been obtained as $L_{1}=0.0836, L_{2}=0$ and $L_{3}=-0.0836$. Also, the maximal Lyapunov exponent of the 3-D novel conservative chaotic system is $L_{1}=0.0836$, which is greater than the maximal Lyapunov exponent of the 3-D NoséHoover conservative chaotic system, viz. $L_{1}=0.014$. The phase portraits of the novel chaotic system were simulated using MATLAB. We also showed that the 3-D novel conservative chaotic system has no equilibrium points and discussed its symmetry and invariance properties. Next, an adaptive controller was designed to achieve generalized projective synchronization (GPS) of two identical novel chaotic systems with unknown system parameters. Generalized projective synchronization is a general type of synchronization which generalizes common types of synchronization such as complete synchronization (CS), anti-synchronization (AS), hybrid synchronization (HS), projective synchronization (PS), etc. The adaptive GPS synchronization result was established using Lyapunov stability theory. Finally, MATLAB simulations were shown to validate and demonstrate the GPS result derived in this work for identical 3-D novel conservative chaotic systems.

\section{References}

1. S.H. Strogatz, Nonlinear dynamics and chaos: With applications to physics, biology, chemistry, and engineering, Perseus Books, Massachusetts, US (1994).

2. E.N. Lorenz, Deterministic nonperiodic flow, Journal of the Atmospheric Sciences, vol. 20, pp. 130-141 (1963).

3. O.E. Rössler, An equation for continuous chaos, Physics Letters A, vol. 57, pp. 397-398 (1976).

4. M.I. Rabinovich and A.L. Fabrikant, Stochastic self-modulation of waves in nonequilibrium media, Sov. Phys. JETP, vol. 50, pp. 311-317, (1979)

5. A. Arneodo, P. Coullet, and C. Tresser, Possible new strange attractors with spiral structure, Communications in Mathematical Physics, vol. 79, pp. 573-579 (1981).

6. J.C. Sprott, Some simple chaotic flows, Physical Review E, vol. 50, pp. 647-650 (1994).

7. G. Chen and T. Ueta, Yet another chaotic oscillator, International Journal of Bifurcation and Chaos, vol. 9, pp. 14651466 (1999).

8. J. Lü and G. Chen, A new chaotic attractor coined, International Journal of Bifurcation and Chaos, vol. 12, pp. 659-661 (2002).

9. R. Shaw, Strange attractors, chaotic behaviour and information flow, Zeitschrift für Naturforschung, vol. 36, pp. 80-112 (1981).

10. B. Feeny and F.C. Moon, Chaos in a forced dry-friction oscillator: Experiments and numerical modeling, Journal of Sound and Vibration, vol. 170, pp. 303-323 (1994).

11. T. Shimizu and N. Moroika, On the bifurcation of a symmetric limit cycle to an asymmetric one in a simple model, Physics Letters A, vol. 76, pp. 201-204 (1980).

12. W. Liu and G. Chen, A new chaotic system and its generation, International Journal of Bifurcation and Chaos, vol. 13, pp. 261267 (2003)

13. G. Cai and Z. Tan, Chaos synchronization of a new chaotic system via nonlinear control, Journal of Uncertain Systems, vol. 1, pp. 235-240 (2007).

14. G. Tigan and D. Opris, Analysis of a 3D chaotic system, Chaos, Solitons and Fractals, vol. 36, pp. 1315-1319 (2008).

15. G.P. Kennedy, Chaos in the Colpitts oscillator, IEEE Transactions on Circuits and Systems-I, vol. 41, pp. 771-774 (1994).
16. J. Wang, D. Lu, and L. Tian, Global synchronization for time delay of WINDMI system, Chaos, Solitons and Fractals, vol. 30, pp. 629-635 (2006).

17. W. Zhou, Y. Xu, H. Lu, and L. Pan, On dynamics analysis of a new chaotic attractor, Physics Letters A, vol. 372, pp. 57735777 (2008).

18. D. Li, A three-scroll chaotic attractor, Physics Letters A, vol. 372, pp. 387-393 (2008).

19. Z. Elhadj, Dynamical analysis of a 3-D chaotic system with only two quadratic nonlinearities, Journal of Systems Science and Complexity, vol. 21, pp. 67-75 (2008).

20. L. Pan, D. $\mathrm{Xu}$, and W. Zhou, Controlling a novel chaotic attractor using linear feedback, Journal of Information and Computing Science, vol. 5, pp. 117-124 (2010).

21. V. Sundarapandian, Analysis and anti-synchronization of a novel chaotic system via active and adaptive controllers, Journal of Engineering Science and Technology Review, vol. 6, pp. 45-52 (2013).

22. F. Yu, C. Wang, Q. Wan, and Y. Hu, Complete switched modified function projective synchronization of a five-term chaotic system with uncertain parameters and disturbances, Pramana, vol. 80, pp. 223-235 (2013).

23. V. Sundarapandian and I. Pehlivan, Analysis, control, synchronization and circuit design of a novel chaotic system, Mathematical and Computer Modelling, vol. 55, pp. 1904-1915 (2012).

24. C. Zhu, Y. Liu, and Y. Guo, Theoretical and numerical study of a new chaotic system, Intelligent Information Management, vol. 2, pp. 104-109 (2010).

25. S. Vaidyanathan, A new six-term 3-D chaotic system with an exponential nonlinearity, Far East Journal of Mathematical Sciences, vol. 79, pp. 135-143 (2013).

26. S. Vaidyanathan, Analysis and adaptive synchronization of two novel chaotic systems with hyperbolic sinusoidal and cosinusoidal nonlinearity and unknown parameters, Journal of Engineering Science and Technology Review, vol. 6, pp. 53-65 (2013).

27. S. Vaidyanathan, A new eight-term 3-D polynomial chaotic system with three quadratic nonlinearities, Far East Journal of Mathematical Sciences, vol. 84, pp. 219-226 (2014). 
28. S. Vaidyanathan, Analysis, control and synchronization of a sixterm novel chaotic system with three quadratic nonlinearities, International Journal of Modelling, Identification and Control, vol. 22, pp. 41-53 (2014).

29. S. Vaidyanathan, Analysis and adaptive synchronization of eight-term 3-D polynomial chaotic systems with three quadratic nonlinearities, European Physical Journal: Special Topics, vol. 223, pp. 1519-1529 (2014).

30. S. Vaidyanathan, Ch. Volos, V.T. Pham, K. Madhavan, and B.A. Idowu, Adaptive backstepping control, synchronization and circuit simulation of a 3-D novel jerk chaotic system with two hyperbolic sinusoidal nonlinearities, Archives of Control Sciences, vol. 24(3), pp. 257-285 (2014).

31. S. Vaidyanathan and K. Madhavan, Analysis, adaptive control and synchronization of a seven-term novel 3-D chaotic system, International Journal of Control Theory and Applications, vol. 6 , pp. 121-137 (2013).

32. I. Pehlivan, I.M. Moroz, and S. Vaidyanathan, Analysis, synchronization and circuit design of a novel butterfly attractor, Journal of Sound and Vibration, vol. 333, pp. 5077-5096 (2014).

33. S. Jafari and J.C. Sprott, Simple chaotic flows with a line equilibrium, Chaos, Solitons and Fractals, vol. 57, pp. 79-84 (2013).

34. V.T. Pham, C. Volos, S. Jafari, Z. Wei and X. Wang, Constructing a novel no-equilibrium chaotic system, International Journal of Bifurcation and Chaos, vol. 24, 1450073 (2014).

35. J.C. Sprott, Elegant Chaos, World Scientific, Singapore, (2010).

36. S. Nosé, Constant temperature molecular dynamics methods, Progress of Theoretical Physics Supplements, vol. 103, pp. 1-46 (1991).

37. H.A. Posch, W.G. Hoover and F.J. Vesely, Canonical dynamics of the Nosé oscillator: stability, order and chaos, Physical Review A, vol. 33(6), pp. 4253-4265 (1986).

38. H.B. Fotsin and J. Daafouz, Adaptive synchronization of uncertain Colpitts oscillators based on parameter identification, Physics Letters A, vol. 339, pp. 304-315 (2005).

39. G.H. Li, S.P. Zhou and K. Yang, Controlling chaos in Colpitts oscillator, Chaos, Solitons and Fractals, vol. 33, pp. 582-587 (2007).

40. A. Sharma, V. Patidar, G. Purohit, and K.K. Sud, Effects on the bifurcation and chaos in forced Duffing oscillator due to nonlinear damping, Communications in Nonlinear Science and Numerical Simulation, vol. 17, pp. 2254-2269 (2012).

41. S. Donati and S.K. Hwang, Chaos and high-level dynamics in coupled lasers and their applications, Progress in Quantum Electronics, vol. 36, pp. 293-341 (2012).

42. I.V. Ermakov, S.T. Kingni, V.Z. Tronciu, and J. Danckaert, Chaotic semiconductor ring lasers subject to optical feedback: Applications to chaos-based communications, Optics Communications, vol. 286, pp. 265-272 (2013).

43. N. Li, W. Pan, L. Yan, B. Luo, and X. Zou, Enhanced chaos synchronization and communication in cascade-coupled semiconductor ring lasers, Communications in Nonlinear Science and Numerical Simulation, vol. 19, pp. 1874-1883 (2014).

44. U. Nehmzow and K. Walker, Quantitative description of robotenvironment interaction using chaos theory, Robotics and Autonomous Systems, vol. 53, pp. 177-193 (2005).

45. S. Iqbal, X. Zang, Y. Zhu, and J. Zhao, Bifurcations and chaos in passive dynamic walking: A review, Robotics and Autonomous Systems, vol. 62, pp. 889-909 (2014).

46. Ch.K. Volos, I.M. Kyprianidis, and I.N. Stouboulos, A chaotic path planning generator for autonomous mobile robots, Robotics and Autonomous Systems, vol. 60, pp. 651-656, (2012).

47. Ch.K. Volos, I.M. Kyprianidis, and I.N. Stouboulos, Experimental investigation on coverage performance of a chaotic autonomous mobile robot, Robotics and Autonomous Systems, vol. 61(12), pp. 1314-1322 (2013).

48. J.C. Roux, Chaos in experimental chemical systems: two examples, North-Holland Mathematics Studies, vol. 103, pp. 345-352 (1985).

49. Y.N. Li, L. Chen, Z.S. Cai, and X.Z. Zhao, Study on chaos synchronization in the Belousov-Zhabotinsky chemical system, Chaos, Solitons and Fractals, vol. 17, pp. 699-707 (2003).

50. Y. Gong, Y. Xie, X. Lin, Y. Hao, and X. Ma, Ordering chaos and synchronization transitions by chemical delay and coupling on scale-free neuronal networks, Chaos, Solitons and Fractals, vol. 43, pp. 96-103 (2010).

51. M. Kyriazis, Applications of chaos theory to the molecular biology of aging, Experimental Gerontology, vol. 26, pp. 569572 (1991).

52. G. Böhm, Protein folding and deterministic chaos: Limits of protein folding simulations and calculations, Chaos, Solitons and Fractals, vol. 1, pp. 375-382 (1991).

53. J.C. Sprott, J.A. Vano, J.C. Wildenberg, M.B. Anderson, and J.K. Noel, Coexistence and chaos in complex ecologies, Physics Letters A, vol. 335, pp. 207-212 (2005).

54. B. Sahoo and S. Poria, The chaos and control of a food chain model supplying additional food to top-predator, Chaos, Solitons and Fractals, vol. 58, pp. 52-64 (2014).

55. T.A. Denton, G.A. Diamond, R.H. Helfant, S. Khan, and H. Karagueuzian, Fascinating rhythm: A primer on chaos theory and its applications to cardiology, American Heart Journal, vol. 120, pp. 1419-1440 (1990).

56. A. Wu, S. Wen, and Z. Zeng, Synchronization control of a class of memristor-based neural networks, Information Sciences, vol. 183, pp. 106-116 (2012).

57. S. Wen, Z. Zeng, and T. Huang, Adaptive synchronization of memristor-based Chua's circuits, Physics Letters A, vol. 376, pp. 2775-2780, (2012).

58. A. Wu, and Z. Zeng, Anti-synchronization control of a class of memristive recurrent neural networks, Communications in Nonlinear Science and Numerical Simulation, vol. 18, pp. 373385 (2013).

59. V.-T. Pham, C. Volos, and L. V. Gambuzza, A memristive hyperchaotic system without equilibrium, Scientific World Journal, vol. 2014, pp. 1-9 (2014).

60. Ch. K. Volos, I. M. Kyprianidis, I. N. Stouboulos, S. G. Stavrinides, A. N. Anagnostopoulos, and M. Ozer, The concept of unidirectionally coupled nonlinear circuits via a memristor, Acta Physica Polonica A, vol. 121, pp. 268-270 (2012).

61. G. He, Z. Cao, P. Zhu, and H. Ogura, Controlling chaos in a chaotic neural network, Neural Networks, vol. 16, pp. 11951200 (2003).

62. E. Kaslik and S. Sivasundaram, Nonlinear dynamics and chaos in fractional-order neural networks, Neural Networks, vol. 32, pp. 245-256 (2012).

63. I.M. Kyprianidis and A.T. Makri, Complex dynamics of FitzHugh-Nagumo type neurons coupled with gap junction under external voltage stimulation, Journal of Engineering Science and Technology Review, vol. 6(4), pp. 104-114 (2013).

64. K. Suzuki and Y. Imai, Decryption characteristics in message modulation type chaos secure communication system using optical fiber ring resonators, Optics Communications, vol. 259, pp. 88-93 (2006).

65. X.Y. Wang and Y.F. Gao, A switch-modulated method for chaos digital secure communication based on user-defined protocol, Communications in Nonlinear Science and Numerical Simulation, vol. 15, pp. 99-104 (2010).

66. O.I. Moskalenko, A.A. Koronovskii, and A.E. Hramov, Generalized synchronization of chaos for secure communication: Remarkable stability to noise, Physics Letters A, vol. 374, pp. 2925-2931 (2010).

67. A. Abdullah, Synchronization and secure communication of uncertain chaotic systems based on full-order and reduced-order output-affine observers, Applied Mathematics and Computation, vol. 219, pp. 10000-10011 (2013).

68. N. Smaoui and A. Kanso, Cryptography with chaos and shadowing, Chaos, Solitons and Fractals, vol. 42, pp. 2312-2321 (2009).

69. R. Rhouma and S. Belghith, Cryptanalysis of a chaos-based cryptosystem on DSP, Communications in Nonlinear Science and Numerical Simulation, vol. 16, pp. 876-884 (2011).

70. Ch K. Volos, I.M. Kyprianidis, and I.N. Stouboulos, Text encryption scheme realized with a chaotic pseudo-random bit generator, Journal of Engineering Science and Technology Review, vol. 6(4), pp. 9-14 (2013).

71. Ch.K. Volos, I.M. Kyprianidis, and I.N. Stouboulos, Image encryption process based on chaotic synchronization phenomena, Signal Processing, vol. 93(5), pp. 1328-1340 (2013).

72. D. Guégan, Chaos in economics and finance, Annual Reviews in Control, vol. 33, pp. 89-93 (2009). 
73. Ch.K. Volos, I.M. Kyprianidis, and I.N. Stouboulos, Synchronization phenomena in coupled nonlinear systems applied in economic cycles, WSEAS Trans. Systems, vol. 11(12), pp. 681-690 (2012).

74. P. Caraiani, Testing for nonlinearity and chaos in economic time series with noise titration, Economics Letters, vol. 120, pp. 192194 (2013).

75. V. Sundarapandian, Output regulation of the Lorenz attractor, Far East Journal of Mathematical Sciences, vol. 42, pp. 289-299 (2010).

76. S. Vaidyanathan, Output regulation of Arneodo-Coullet chaotic system, Communications in Computer and Information Science, vol. 131, pp. 585-593 (2011).

77. S. Vaidyanathan, Output regulation of the unified chaotic system, Communications in Computer and Information Science, vol. 198, pp. 1-9 (2011).

78. S. Vaidyanathan, Output regulation of the Liu chaotic system, Applied Mechanics and Materials, vols. 110-116, pp. 3982-3989 (2012).

79. G. Chen, A simple adaptive feedback control method for chaos and hyper-chaos control, Applied Mathematics and Computation, vol. 217, pp. 7258-7264 (2011).

80. J. Zheng, A simple universal adaptive feedback controller for chaos and hyperchaos control, Computers \& Mathematics with Applications, vol. 61, pp. 2000-2004 (2011).

81. S. Vaidyanathan, Adaptive controller and synchronizer design for the Qi-Chen chaotic system, Lecture Notes of the Institute for Computer Sciences, Social-Informatics and Telecommunications Engineering, vol. 85, pp. 124-133 (2012).

82. V. Sundarapandian, Adaptive control and synchronization design for the Lu-Xiao chaotic system, Lecture Notes in Electrical Engineering, vol. 131, pp. 319-327 (2013).

83. S. Vaidyanathan, A ten-term novel 4-D hyperchaotic system with three quadratic nonlinearities and its control, International Journal of Control Theory and Applications, vol. 6, pp. 97-109 (2013).

84. S. Vaidyanathan, Analysis, control and synchronization of hyperchaotic Zhou system via adaptive control, Advances in Intelligent Systems and Computing, vol. 177, pp. 1-10 (2013).

85. D. Yang and J. Zhou, Connections among several chaos feedback control approaches and chaotic vibration control of mechanical systems, Communications in Nonlinear Science and Numerical Simulation, vol. 19, pp. 3954-3968 (2014).

86. M.T. Yassen, Chaos control of chaotic dynamical systems using backstepping design, Chaos, Solitons and Fractals, vol. 27, pp. 537-548 (2006).

87. J.A. Laoye, U.E. Vincent, and S.O. Kareem, Chaos control of 4D chaotic systems using recursive backstepping nonlinear controller, Chaos, Solitons and Fractals, vol. 39, pp. 356-362 (2009).

88. D. Lin, X. Wang, F. Nian, and Y. Zhang, Dynamic fuzzy neural networks modeling and adaptive backstepping tracking control of uncertain chaotic systems, Neurocomputing, vol. 73, pp. 2873-2881 (2010)

89. S. Vaidyanathan, Sliding mode control based global chaos control of Liu-Liu-Liu-Su chaotic system, International Journal of Control Theory and Applications, vol. 5, pp. 15-20 (2012).

90. S. Vaidyanathan, Global chaos control of hyperchaotic Liu system via sliding mode control, vol. 5, pp. 117-123 (2012).

91. L. Kocarev and U. Parlitz, General approach for chaos synchronization with applications to communications, Physical Review Letters, vol. 74, pp. 5028-5030 (1995).

92. K. Murali and M. Lakshmanan, Secure communication using a compound signal using sampled-data feedback, Applied Mathematics and Mechanics, vol. 11, pp. 1309-1315, (1995).

93. M. Feki, An adaptive chaos synchronization scheme applied to secure communication, Chaos, Solitons and Fractals, vol. 18, pp. 141-148 (2003).

94. J. Yang and F. Zhu, Synchronization for chaotic systems and chaos-based secure communications via both reduced-order and step-by-step sliding mode observers, Communications in Nonlinear Science and Numerical Simulation, vol. 18, pp. 926937 (2013).

95. L. Kocarev, Chaos-based cryptography: a brief overview, IEEE Circuits and Systems, vol. 1, pp. 6-21 (2001).

96. H. Gao, Y. Zhang, S. Liang and D. Li, A new chaotic algorithm for image encryption, Chaos, Solitons and Fractals, vol. 29, pp. 393-399 (2006).
97. Y. Wang, K.W. Wang, X. Liao and G. Chen, A new chaos-based fast image encryption, Applied Soft Computing, vol. 11, pp. 514-522, (2011).

98. Ch. K. Volos, I. M. Kyprianidis, and I. N. Stouboulos, Image encryption process based on chaotic synchronization phenomena, Signal Processing, vol. 93(5), pp. 1328-1340 (2013).

99. Ch. K. Volos, I. M. Kyprianidis, and I. N. Stouboulos, Text encryption scheme realized with a chaotic pseudo-random bit generator, Journal of Engineering Science and Technology Review, vol. 6(4), pp. 9-14 (2013).

100. L.M. Pecora and T.L. Carroll, Synchronization in chaotic systems, vol. 64, pp. 821-825 (1990).

101. S. Vaidyanathan and S. Rasappan, New results on the global chaos synchronization for Liu-Chen-Liu and Lü chaotic systems, Communications in Computer and Information Science, vol. 102, pp. 20-27 (2010).

102. S. Vaidyanathan and S. Rasappan, Hybrid synchronization of hyperchaotic Qi and Lü systems by nonlinear control, Communications in Computer and Information Science, vol. 131, pp. 585-593 (2011).

103. S. Vaidyanathan and K. Rajagopal, Anti-synchronization of Li and $\mathrm{T}$ chaotic systems by active nonlinear control, Communications in Computer and Information Science, vol. 198, pp. 175-184 (2011).

104. S. Vaidyanathan and S. Rasappan, Global chaos synchronization of hyperchaotic Bao and $\mathrm{Xu}$ systems by active nonlinear control, Communications in Computer and Information Science, vol. 198, pp. 10-17 (2011).

105. S. Vaidyanathan and K. Rajagopal, Global chaos synchronization of hyperchaotic Pang and Wang systems by active nonlinear control, Communications in Computer and Information Science, vol. 204, pp. 84-93 (2011).

106. S. Vaidyanathan, Hybrid chaos synchronization of Liu and Lü systems by active nonlinear control, Communications in Computer and Information Science, vol. 204, pp. 1-10 (2011).

107. P. Sarasu and V. Sundarapandian, Active controller design for generalized projective synchronization of four-scroll chaotic systems, International Journal of Systems Signal Control and Engineering Application, vol. 4, pp. 26-33 (2011).

108. S. Vaidyanathan and K. Rajagopal, Hybrid synchronization of hyperchaotic Wang-Chen and hyperchaotic Lorenz systems by active non-linear control, International Journal of Systems Signal Control and Engineering Application, vol. 4, pp. 55-61 (2011).

109. S. Pakiriswamy and S. Vaidyanathan, Generalized projective synchronization of three-scroll chaotic systems via active control, Lecture Notes of the Institute for Computer Sciences, Social-Informatics and Telecommunications Engineering, vol. 85, pp. 146-155 (2012).

110. V. Sundarapandian and R. Karthikeyan, Hybrid synchronization of hyperchaotic Lorenz and hyperchaotic Chen systems via active control, Journal of Engineering and Applied Sciences, vol. 7, pp. 254-264 (2012).

111. R. Karthikeyan and V. Sundarapandian, Hybrid chaos synchronization of four-scroll systems via active control, Journal of Electrical Engineering, vol. 65, pp. 97-103 (2014).

112. E.M. Shahverdiev and K.A. Shore, Impact of modulated multiple optical feedback time delays on laser diode chaos synchronization, Optics Communications, vol. 282, pp. 35683572 (2009).

113. T. Botmart, P. Niamsup, and X. Liu, Synchronization of nonautonomous chaotic systems with time-varying delay via delayed feedback control, Communications in Nonlinear Science and Numerical Simulation, vol. 17, pp. 1894-1907 (2012).

114. S. Bowong, Adaptive synchronization between two different chaotic dynamical systems, Communications in Nonlinear Science and Numerical Simulation, vol. 12, pp. 976-985 (2007).

115. W. Lin, Adaptive chaos control and synchronization in only locally Lipschitz systems, Physics Letters A, vol. 372, pp. 31953200 (2008).

116. H. Salarieh and A. Alasty, Adaptive chaos synchronization in Chua's systems with noisy parameters, Mathematics and Computers in Simulation, vol. 79, pp. 233-241 (2008).

117. H. Salarieh and A. Alasty, Adaptive synchronization of two chaotic systems with stochastic unknown parameters, Communications in Nonlinear Science and Numerical Simulation, vol. 14, pp. 508-519 (2009). 
118. S. Vaidyanathan and K. Rajagopal, Global chaos synchronization of Lü and Pan systems by adaptive nonlinear control, Communications in Computer and Information Science, vol. 205, pp. 193-202 (2011).

119. V. Sundarapandian and R. Karthikeyan, Anti-synchronization of Lü and Pan chaotic systems by adaptive nonlinear control, European Journal of Scientific Research, vol. 64, pp. 94-106 (2011).

120. V. Sundarapandian and R. Karthikeyan, Anti-synchronization of hyperchaotic Lorenz and hyperchaotic Chen systems by adaptive control, International Journal of Systems Signal Control and Engineering Application, vol. 4, pp. 18-25 (2011).

121. V. Sundarapandian and R. Karthikeyan, Adaptive antisynchronization of uncertain Tigan and Li systems, Journal of Engineering and Applied Sciences, vol. 7, pp. 45-52 (2012).

122. P. Sarasu and V. Sundarapandian, Generalized projective synchronization of three-scroll chaotic systems via adaptive control, European Journal of Scientific Research, vol. 72, pp. 504-522 (2012).

123. P. Sarasu and V. Sundarapandian, Generalized projective synchronization of two-scroll systems via adaptive control, International Journal of Soft Computing, vol. 7, pp. 146-156 (2012).

124. P. Sarasu and V. Sundarapandian, Adaptive controller design for the generalized projective synchronization of 4-scroll systems, International Journal of Systems Signal Control and Engineering Application, vol. 5, pp. 21-30 (2012).

125. S. Vaidyanathan and K. Rajagopal, Global chaos synchronization of hyperchaotic Pang and hyperchaotic Wang systems via adaptive control, International Journal of Soft Computing, vol. 7, pp. 28-37 (2012).

126. S.H. Lee, V. Kapila, M. Porfiri, and A. Panda, Master-slave synchronization of continuously and intermittently coupled sampled-data chaotic oscillators, Communications in Nonlinear Science and Numerical Simulation, vol. 15, pp. 4100-4113 (2010).

127. X.Z. Jin and J.H. Park, Adaptive synchronization for a class of faulty and sampling coupled networks with its circuit implement, Journal of the Franklin Institute, vol. 351, pp. 4317-4333 (2014).

128. C.K. Zhang, L. Jiang, Y. He, Q.H. Wu and M. Wu, Asymptotical synchronization for chaotic Lur'e systems using sampled-data control, Communications in Nonlinear Science and Numerical Simulation, vol. 18, pp. 2743-2751 (2013).

129. X. Xiao, L. Zhou, and Z. Zhang, Synchronization of chaotic Lur'e systems with quantized sampled-data controller, Communications in Nonlinear Science and Numerical Simulation, vol. 19, pp. 2039-2047 (2014).

130. S. Rasappan and S. Vaidyanathan, Global chaos synchronization of WINDMI and Coullet chaotic systems by backstepping control, Far East Journal of Mathematical Sciences, vol. 67, pp. 265-287 (2012).
131. S. Rasappan and S. Vaidyanathan, Synchronization of hyperchaotic Liu system via backstepping control with recursive feedback, Communications in Computer and Information Science, vol. 305, pp. 212-221 (2012).

132. S. Rasappan and S. Vaidyanathan, Hybrid synchronization of nscroll Chua and Lur'e chaotic systems via backstepping control with novel feedback, Archives of Control Sciences, vol. 22, pp. 343-365 (2012).

133. R. Suresh and V. Sundarapandian, Global chaos synchronization of a family of n-scroll hyperchaotic Chua circuits using backstepping control with recursive feedback, Far East Journal of Mathematical Sciences, vol. 73, pp. 73-95 (2013).

134. S. Rasappan and S. Vaidyanathan, Hybrid synchronization of n-scroll chaotic Chua circuits using adaptive backstepping control design with recursive feedback, Malaysian Journal of Mathematical Sciences, vol. 7, pp. 219-246 (2013).

135. S. Vaidyanathan and S. Rasappan, Global chaos synchronization of n-scroll Chua circuit and Lur'e system using backstepping control design with recursive feedback, Arabian Journal for Science and Engineering, vol. 39, pp. 3351-3364 (2014).

136. S. Rasappan and S. Vaidyanathan, Global chaos synchronization of WINDMI and Coullet chaotic systems using adaptive backstepping control design, Kyungpook Mathematical Journal, vol. 54, pp. 293-320 (2014).

137. H.T. Yau, Chaos synchronization of two uncertain chaotic nonlinear gyros using fuzzy sliding mode control, Mechanical Systems and Signal Processing, vol. 22, pp. 408-418 (2008).

138. H. Li, X. Liao, C. Li, and C. Li, Chaos control and synchronization via a novel chatter free sliding mode control strategy, Neurocomputing, vol. 74, pp. 3212-3222 (2012).

139. S. Vaidyanathan and S. Sampath, Global chaos synchronization of hyperchaotic Lorenz systems by sliding mode control, Communications in Computer and Information Science, vol. 205, pp. 156-164 (2011).

140. V. Sundarapandian and S. Sivaperumal, Sliding controller design of hybrid synchronization of four-wing chaotic systems, International Journal of Soft Computing, vol. 6, pp. 224-231 (2011).

141. S. Vaidyanathan and S. Sampath, Anti-synchronization of fourwing chaotic systems via sliding mode control, International Journal of Automation and Computing, vol. 9, pp. 274-279 (2012).

142. H.T. Yau, Chaos synchronization of two uncertain chaotic nonlinear gyros using fuzzy sliding mode control, Mechanical Systems and Signal Processing, vol. 22(2), pp. 408-418 (2013).

143. S. Vaidyanathan, Global chaos synchronisation of identical LiWu chaotic systems via sliding mode control, International Journal of Modelling, Identification and Control, vol. 22, no. 2, pp. 170-177 (2014).

144. H.K. Khalil, Nonlinear System, $3^{\text {rd }}$ ed., Prentice Hall, New Jersey, USA (2002). 